\title{
Principal component analysis for nonlinear model reference adaptive control
}

\author{
Richard B. McLain, Michael A. Henson* \\ Department of Chemical Engineering, Louisiana State University, Baton Rouge, LA 70803-7303, USA
}

Received 17 June 1998; received in revised form 1 March 2000; accepted 1 March 2000

\begin{abstract}
A nonlinear adaptive control strategy based on radial basis function networks and principal component analysis is presented. The proposed method is well suited for low dimensional nonlinear systems that are difficult to model and control via conventional means. The effective system dimension is reduced by applying nonlinear principal component analysis to state variable data obtained from open-loop tests. This allows the radial basis functions to be placed in a lower dimensional space than the original state space. The total number of basis functions is specified a priori, and an algorithm which adjusts the location of the basis function centers to surround the current operating point is presented. The basis function weights are adapted on-line such that the plant output asymptotically tracks a linear reference model. A highly nonlinear polymerization reactor is used to compare the nonlinear adaptive controller to a linear state feedback controller that utilizes the same amount of plant information. (C) 2000 Elsevier Science Ltd. All rights reserved.
\end{abstract}

Keywords: Nonlinear systems; Radial basis function networks; Model reference adaptive control; Nonlinear principal component analysis; Polymerization reactor control

\section{Introduction}

The application of nonlinear process control techniques is limited by a lack of accurate nonlinear dynamic models. Fundamental models can be difficult to derive due to the lack of fundamental process knowledge and the limited availability of experimental data for determining unknown parameters. While a variety of nonlinear system identification techniques have been proposed, there are many unresolved issues concerning model structure and data requirements (Pearson \& Ogunnaike, 1997). An alternative to the model-based controller design approach is to adaptively construct the nonlinear controller on-line without an explicit dynamic model. By analogy to the linear case (Sastry \& Bodson, 1989), this is called direct adaptive nonlinear control. The most popular approach is to adapt the controller parameters such that the plant output asymptotically tracks the output of a linear reference model (Sastry \& Isidori, 1989). This is termed nonlinear model reference adaptive control (MRAC).

* Corresponding author. Fax: + 1-225-3881476.

E-mail address: henson@che.lsu.edu (M.A. Henson)
We have developed a nonlinear MRAC strategy based on radial basis function (RBF) networks for minimum phase nonlinear systems (McLain \& Henson, 1997; McLain, Henson \& Pottmann, 1999). The only information required about the plant is the relative degree, the sign of a Lie derivative which appears in the associated input-output linearizing control law, and measurements of the state variables. Unknown controller functions are constructed on-line using RBF networks such that the plant output tracks the output of a linear reference model. Potential RBF centers are placed on a regular grid in the state space, and a particular basis function is activated only if the closedloop system evolves 'near' its center. Due to the large number of adjustable parameters in the RBF networks, this placement scheme is computationally tractable only for low-dimensional systems with small operating regimes.

In this paper, nonlinear principal component analysis (NPCA) is investigated as a means to extend the nonlinear MRAC strategy to higher dimensional systems. NPCA is used to reduce the effective system dimension so that RBF centers can be placed in a lower dimen- 
sional space which reflects the most critical directions of dynamic operation. Computational efficiency is further enhanced by fixing the total number of basis functions and utilizing an algorithm for on-line addition/pruning of RBF centers. The proposed modifications allow the MRAC strategy to be applied to nonlinear systems where the critical dynamic behavior can be represented in two or (possibly) three dimensions. The nonlinear MRAC controller is compared to a linear state feedback controller using a highly nonlinear polymerization reactor model.

The remainder of this paper is organized as follows. In Section 2, the basic nonlinear MRAC strategy is presented. On-line computational issues associated with the method are discussed in Section 3. Dimensionality reduction with NPCA is discussed in Section 3.1. In Section 3.2, an efficient method for on-line placement of $\mathrm{RBF}$ centers in the reduced dimensional space is presented. In Section 4, a highly nonlinear polymerization reactor model is used to evaluate the proposed computational enhancements. Finally, a summary and conclusions are presented in Section 5.

\section{Basic nonlinear MRAC strategy}

Consider a single-input, single-output nonlinear system of the form,

$\dot{x}=f(x)+g(x) u$

$y=h(x)$

where $x$ is a $n$ dimensional vector of state variables, $u$ is a scalar manipulated input, $y$ is a scalar controlled output, and $f(x) \quad g(x)$ and $h(x)$ are nonlinear functions. The MRAC strategy is applicable to nonlinear systems (1) with the following properties:

1. The system dimension is small $(n \leq 2)$. As discussed in Section 3, the on-line adaptation scheme becomes intractable for higher dimensional systems.

2. The nonlinear functions $f(x)$ and $g(x)$ are unknown, while the nonlinear function $h(x)$ is known. This assumption is reasonable because $h(x)$ is chosen as part of the control system design.

3 . The state vector $(x)$ is measured. This is a restrictive assumption that will be relaxed in the simulation study presented in Section 4.

4. The relative degree is well-defined and the zero dynamics are stable. These restrictions are required because the controller design procedure is based on input-output linearization.

5. The sign of a Lie derivative that appears in the associated input-output linearizing control law is known. This condition can be viewed as a generalization of the high frequency gain condition in linear adaptive control theory (Sastry \& Bodson, 1989).

The goal of this paper is to extend the applicability of the nonlinear MRAC strategy by relaxing the first condition. The other conditions remain as standing assumptions.

The control objective is to make the controlled output $(y)$ asymptotically track the output of a linear reference model $\left(y_{m}\right)$. The following reference model is appropriate for nonlinear systems of relative degree one (Isidori, 1989),

$\dot{y}_{m}=-\gamma y_{m}+\gamma r$

where $r$ is the setpoint and $\gamma>0$ is a controller tuning parameter that determines the speed of response. In this paper, we focus on the relative degree one case. However, the following development can be extended to higher relative degree systems using the filtered regressor approach (Sanner \& Slotine, 1992; McLain et al., 1999). The input-output linearizing control law that achieves the model matching objective is (Isidori, 1989),

$u=\frac{-L_{f} h(x)-\gamma h(x)+\gamma r}{L_{g} h(x)} \equiv \frac{-\alpha^{*}(x)+\gamma r}{\beta^{*}(x)}$

where $L_{f} h(x)$ and $L_{g} h(x)$ are Lie derivatives. Note that the nonlinear functions $f(x), g(x)$ and $h(x)$ must be known to construct the Lie derivatives.

The linear approximation of (1) about a steady-state operating point $(\bar{x}, \bar{u}, \bar{y})$ can be written as,

$\dot{x}^{\prime}=A x^{\prime}+b u^{\prime}$

$y^{\prime}=c x^{\prime}$

where: $x^{\prime}, u^{\prime}$ and $r^{\prime}$ are deviation variables; and $A, b$ and $c$ are state-space matrices of the linear model. The matrices $A, b$ and $c$ can be obtained via standard linear system identification techniques (Ljung, 1989). The linear state feedback controller that provides local model matching with respect to the reference model (2) is,

$u^{\prime}=\frac{-c A x^{\prime}-\gamma c x^{\prime}+\gamma r^{\prime}}{c b} \equiv \frac{-k_{1} x^{\prime}+\gamma r^{\prime}}{k_{2}}$

where $k_{1}$ and $k_{2}$ are linear controller gains. As discussed below, the transient performance of the nonlinear MRAC controller can be improved by embedding the linear controller gains within the nonlinear controller functions.

The nonlinear control law (3) cannot be constructed if the model functions $f(x)$ and $g(x)$ are unknown. The most common solution to this problem is to generate estimates of the unknown model functions via nonlinear system identification (Pearson \& Ogunnaike, 1997) and then use these estimates to construct the nonlinear controller functions. However, there are a number of unresolved issues that limit the applicability of nonlinear system identification tecnhiques including: 
1. Selection of an appropriate model structure.

2. Input sequence design for generating plant data.

3. Robust estimation of model parameters from plant data.

Additional complications appear when radial basis function (RBF) networks are used to estimate the unknown functions of a nonlinear state-space model. The number of estimated functions can be as large as $2 n$ where $n$ is the dimension of the nonlinear system. This dimensionality problem hinders the development of indirect adaptive control schemes (Polycarpou \& Ioannou, 1991; Pottmann \& Henson, 1997).

In many chemical process applications, the dynamic model is developed for controller design rather than to enhance fundamental process understanding. This observation motivates the direct approximation of the unknown controller functions $\alpha^{*}(x)$ and $\beta^{*}(x)$. As compared to indirect adaptive control, an important advantage of the direct approach is that only the nonlinear functions that actually appear in the nonlinear control law need to be approximated. As discussed below, a maximum of two controller functions must be generated for single-input, single-output systems.

The controller functions are approximated by rewriting the control law (3) as:

$\alpha^{*}(x)+\beta^{*}(x) u=\gamma r$

Taylor series expansions of the controller functions and subsequent simplification yields (McLain \& Henson, 1997),

$\left[k_{1} x^{\prime}+\tilde{\alpha}\left(x^{\prime}\right)\right]+\left[k_{2}+\tilde{\beta}\left(x^{\prime}\right)\right] u^{\prime}=\gamma r^{\prime}$

where: $k_{1}$ and $k_{2}$ are the linear controller gains in (5); $\tilde{\alpha}\left(x^{\prime}\right)$ represents second-order and higher terms in $\alpha^{*}(x)$ and second-order and higher term in $\beta^{*}(x)$ that are independent of the input; and $\widetilde{\beta}\left(x^{\prime}\right)$ represents first-order and higher terms in $\beta^{*}(x)$ that depend on the input. Thus, the input-output linearizing control law (3) has the following deviation form:

$u^{\prime}=\frac{-\left[k_{1} x^{\prime}+\tilde{\alpha}\left(x^{\prime}\right)\right]+\gamma r^{\prime}}{k_{2}+\tilde{\beta}\left(x^{\prime}\right)}$

The nonlinear controller design problem is reduced to approximating the higher-order functions $\tilde{\alpha}\left(x^{\prime}\right)$ and $\widetilde{\beta}\left(x^{\prime}\right)$. We assume the functions can be represented as,

$\tilde{\alpha}\left(x^{\prime}\right)=\sum_{i=1}^{N} \alpha_{i}^{*} \phi_{i}\left(x^{\prime}\right)=\alpha^{* T} \phi\left(x^{\prime}\right)$

$\tilde{\beta}\left(x^{\prime}\right)=\sum_{i=1}^{N} \beta_{i}^{*} \phi_{i}\left(x^{\prime}\right)=\beta^{* T} \phi\left(x^{\prime}\right)$

where: $\alpha^{*}$ and $\beta^{*}$ are vectors of unknown, constant parameters; $\phi\left(x^{\prime}\right)$ is a vector of radial basis functions (RBFs); and $N$ is the number of basis functions. In practice, the relations (9) will hold only in an approximate sense. An implementable control law is obtained by replacing the unknown controller parameters with time-varying controller parameters $\alpha(t)$ and $\beta(t)$,

$u^{\prime}=\frac{-\left[k_{1} x^{\prime}+\alpha^{T} \phi\left(x^{\prime}\right)\right]+\gamma r^{\prime}}{k_{2}+\beta^{T} \phi\left(x^{\prime}\right)}$

where we assume $k_{2}+\beta^{T} \phi\left(x^{\prime}\right) \neq 0$ to ensure the control law remains well defined. Because the sign of $\beta^{*}(x)$ is known by assumption, this condition usually can be satisfied by initializing the controller with $\beta(0)=0$.

Update laws for the controller parameters $\alpha(t)$ and $\beta(t)$ are derived via Lyapunov stability analysis. It can be shown that the tracking error $e \equiv y_{m}-y$ has the dynamics (McLain \& Henson, 1997),

$\dot{e}=-\gamma e+\Psi_{1}^{T} \phi\left(x^{\prime}\right)+\Psi_{2}^{T} \phi\left(x^{\prime}\right) u^{\prime}$

where $\Psi_{1}=\alpha-\alpha^{*}$ and $\Psi_{2}=\beta-\beta^{*}$ are parameter error vectors. This error equation suggests the following gradient update laws (Sastry \& Bodson, 1989),

$\dot{\Psi}_{1}=\dot{\alpha}=-\eta_{1} e \phi\left(x^{\prime}\right)$

$\dot{\Psi}_{2}=\dot{\beta}=-\eta_{2} e \phi\left(x^{\prime}\right) u^{\prime}$

where $\eta_{i}>0$ are adjustable gains. It is important to note that the nonlinear control law (3) does not include an explicit integral term because the parameter update laws provide implicit integral action for offset-free tracking. Closed-loop stability is proven using the Lyapunov function (McLain et al., 1999):

$V=\frac{e^{2}}{2}+\frac{1}{2 \eta_{1}} \Psi_{1}^{T} \Psi_{1}+\frac{1}{2 \eta_{2}} \Psi_{2}^{T} \Psi_{2}$

\section{Computational enhancements}

The key feature of the nonlinear MRAC strategy is that RBFs centered in the $n$ dimensional state space are used to generate approximations of the unknown controller functions. In theory, RBFs must be placed throughout the entire state space since trajectories of the closed-loop system cannot be predicted a priori. Clearly this scheme cannot be implemented because the estimation problem is computationally intractable.

We have addressed this problem by placing potential locations for RBF centers on a regular grid in the state space (McLain et al., 1999). A particular RBF is activated only if the closed-loop system evolves 'near' its center. A disadvantage of this placement scheme is that a very large number of RBFs may be activated if the system dimension is large and/or the process operates in multiple regions (e.g. a polymerization reactor with different steady-state operating conditions). The addition of each RBF introduces two adjustable parameters $\left(\alpha_{i}, \beta_{i}\right)$ and two differential equations for the associated gradient update laws (12). The total number of differen- 
tial equations is $2 N$ where $N$ is the number of active basis functions. Real-time application is feasible only for low-dimensional systems $(n \leq 2)$ because the number of basis functions required to cover the operating region increases as the power of the system dimension. It is desirable to minimize the total number of adjustable controller parameters to facilitate on-line implementation.

In this paper, the nonlinear MRAC strategy is extended to higher dimensional systems by developing two enhancements of the RBF center placement scheme. The first enhancement involves the use of nonlinear principal component analysis (NPCA) to generate a reduced dimensional space for nonlinear function approximation. The objective is to decrease the number of adjustable parameters by placing the RBFs in the reduced dimensional space. The second enhancement is the development of an efficient addition/pruning algorithm that avoids the computational problems associated with the continuous addition of RBFs. The objective is to limit the maximum number of active basis functions in the reduced dimensional space.

\subsection{Dimensionality reduction via NPCA}

The objective is to construct a reduced dimensional space $Z$ that provides a more efficient representation of the process dynamics than the original state space $X$. The first step is to determine a $Z$ space dimension that yields an acceptable compromise between prediction accuracy and computational efficiency. The second step is to determine coordinates for the $Z$ space that minimize the amount of lost information. In practice, these problems must be solved simultaneously by developing the required mappings for several dimensions and then analyzing the results. As discussed below, we use the sum of squared errors (SSE) between the actual state variables and the estimated state variables obtained from the derived mappings as a measure of prediction accuracy. It is important to emphasize that the nonlinear MRAC stategy is restricted to nonlinear systems that yield a $Z$ space with a maximum of three dimensions. Otherwise, the on-line estimation problem becomes intractable.

Principal component analysis (PCA) is a linear technique for mapping a multi-dimensional data set into a lower dimension space while minimizing the loss of information (Kresta, MacGregor \& Marlin, 1991). The basic idea is to project the original space onto a lower dimensional linear subspace spanned by the eigenvectors of the covariance matrix corresponding to the largest eigenvalues. The reader is referred elsewhere (Mardia, Kent \& Bibby, 1980) for a review of linear PCA.

Several investigators have attempted to extend the PCA approach to nonlinear mappings. The principal curves method (Hastie \& Stuetzle, 1989) minimizes a distance property similar to PCA but the linear subspace assumption is relaxed. The principal curves method can produce a projection that is discontinuous. While this characteristic may be advantageous in a theoretical setting, it is not desirable when the intended application is real-time control. More importantly, each data point only generates an associated score. That is, the algorithm does not produce an explicit nonlinear principal component model. Consequently, it is not possible to develop process estimation and control techniques based on this method (Dong \& McAvoy, 1994).

Kramer (1991) has proposed a five-layer, auto-associative neural network for nonlinear principal component analysis (NPCA). The second and fourth layers contain sigmoidal nodes, while linear nodes are used in the other three layers. The networks maps the full dimensional data into a reduced dimensional space and then performs an expansion to produce estimates of the original data. The backpropagation algorithm is used to train the network to perform the identity mapping. The trained network provides the necessary mappings to transform data between the full and reduced dimensional spaces. This method has been shown to represent data with fewer dimensions and greater accuracy than PCA (Kramer, 1991).

Tan and Mavrovouniotis (1995) have proposed a nonlinear dimensionality reduction technique based on optimization of the neural network inputs. The basic idea is to reduce the five-layer auto-associative network to a three layer network by simultaneously optimizing the network parameters and the network inputs to reproduce the corresponding output vectors. Unfortunately, input vectors are known only for the output vectors used for network training. For other data sets, the input vector must be optimized for each output of interest while holding the network parameters constant. Because this method is not amenable to on-line implementation, we utilize the NPCA method of Kramer (1991) in this study.

The five-layer NPCA network used for the polymerization reactor example in Section 4 is shown in Fig. 1. The network maps vectors from the original $X$ space to the reduced dimensional $Z$ space, and then maps vectors from the $Z$ space to a new space $\hat{X}$ that represents an approximation of the $X$ space. The first layer of the network simply distributes the $x$ vector to nodes in the first hidden layer. The third layer produces the $z$ vector from the outputs of the first hidden layer. The $z$ vector is distributed to a second hidden layer, and the outputs from this layer serve as the inputs for the fifth layer from which the estimated state vector $(\hat{x})$ is produced. The network is trained to perform the identity mapping (Kramer, 1991). The resulting network provides a mapping model $z=\varphi(x)$ and a demapping model $\hat{x}=\xi(z)$. As discussed below, the modified nonlinear MRAC 
strategy utilizes the mapping model to transform state measurements from the $X$ space into vectors in the $Z$ space.

The nonlinear controller is constructed in the reduced dimensional space $Z$ such that the controlled output tracks the output of a linear reference model. Selection of an appropriate reference model is difficult because the relative degree of the nonlinear system in the $Z$ space is unknown. The simplest choice is the first-order reference model (2). More complicated reference models can be used if appropriate, but the resulting parameter update laws are more dificult to implement (McLain et al., 1999).

We assume there exists a nonlinear control law that achieves the model matching objective in the reduced space $Z$ :

$u=\frac{-\alpha^{*}(z)+\gamma r}{\beta^{*}(z)}$

This is a reasonable simplifying assumption if the reduced space provides a good approximation of the process dynamics in the original state space. It is important to emphasize that this assumption is invoked only to derive the parameter updates laws. An implementable version of the nonlinear control law is obtained by approximating the unknown controller functions using RBF networks,

$u^{\prime}=\frac{-\left[k_{1} z^{\prime}+\alpha^{T} \phi\left(z^{\prime}\right)\right]+\gamma r^{\prime}}{k_{2}+\beta^{T} \phi\left(z^{\prime}\right)}$

where $z^{\prime}, u^{\prime}$ and $r^{\prime}$ are deviation variables; $\alpha$ and $\beta$ are vectors of adjustable controller parameters; and $k_{1}$ and $k_{2}$ are linear controller gains chosen to provide local tracking of the reference model. The linear gains are computed as in (5) using an empirical linear model constructed in the $Z$ space:

$k_{1}=c A+\gamma c, \quad k_{2}=c b$

As discussed in Section 4, the linear model is derived using the mapping model $z=\varphi(x)$, state variable data for small amplitude input changes around the nominal operating point, and standard linear system identification techniques (Ljung, 1989). By analogy to the full dimensional problem, the appropriate parameter update laws are,

$\dot{\alpha}=-\eta_{1} e \phi\left(z^{\prime}\right)$

$\dot{\beta}=-\eta_{2} e \phi\left(z^{\prime}\right) u^{\prime}$

where: $e \equiv y_{m}-y$ and $\eta_{1}$ and $\eta_{2}$ are positive adaptation gains. It is important to note that the stability arguments used in the full dimensional problem are not applicable to reduced dimensional case.

\subsection{RBF center placement}

The proposed NPCA method facilitates the application of the nonlinear MRAC strategy to higher dimensional systems. Even with this computational enhancement, the RBF center placement scheme utilized in our previous work (Pottmann \& Henson, 1997; McLain et al., 1999) may cause the parameter estimation problem to become intractable because the number of active basis functions grows with time. We address this problem by fixing the total number $(N)$ of active RBFs in the network. Potential locations for RBF centers are placed on a regular grid in the reduced dimensional space $Z$. Guidelines for selecting an appropriate mesh size based on smoothness properties of the approximated functions are discussed elsewhere (Sanner \& Slotine, 1992).

In this paper, we utilize a locally supported RBF of the form (Pottmann \& Henson, 1997),

$\phi(r)= \begin{cases}(1-r)^{4}\left(1+4 r+3 r^{2}+0.75 r^{3}\right) & r \in[0,1] \\ 0 & \text { elsewhere }\end{cases}$

$r^{2}=\sum_{j=1}^{n} \frac{\left(x_{j}-x_{c}\right)^{2}}{a_{j}^{2}}$

where $n$ is the state dimension, $x_{c}$ is the basis function center, and the $a_{j}$ are scaling parameters. The function is said to have compact support because it is identically zero outside a compact subset of $R$. The locally supported basis function (18) is well suited for adaptive control applications because only a subset of the functions need to be updated at any particular time. The scaling parameters are chosen to fix the coverage of a single RBF. This allows the maximum number of basis functions $(N)$ that can be active to be determined.

A particular basis function is activated only if the closed-loop system evolves 'near' its center. The total number of active functions is held constant by pruning RBFs that are not centered near the current operating point. New centers are added and old centers are pruned as the nonlinear system transitions through the reduced space $Z$. We have found this algorithm to be simpler and more effective than RBF center placement techniques based on recursive clustering (Chen, Billings \& Grant, 1992). Another advantage of the proposed scheme is that the controller parameters can be initialized as $\alpha(0)=$ $\beta(0)=0$ since $\mathrm{RBF}$ centers being added and pruned have small contributions at the current operating point.

\section{Simulation study}

\subsection{Polymerization reactor model}

The modified nonlinear MRAC strategy is evaluated using a four dimensional polymerization reactor model. 
Table 1

Nominal operating conditions

\begin{tabular}{lcll}
\hline Variable & Value & Variable & Value \\
\hline$D_{a p}$ & $5.871 \times 10^{6}$ & $\gamma_{p}$ & 6.846 \\
$D_{a d}$ & $3.6447 \times 10^{11}$ & $\gamma_{d}$ & 6.892 \\
$\beta$ & 1.3 & $B$ & 0.3635 \\
$W$ & $1.013 \times 10^{-7}$ & $x_{2 c}$ & 0 \\
$x_{1}$ & 1.206 & $x_{2}$ & 0.08653 \\
$x_{3}$ & 0.01424 & $x_{4}$ & 1.865 \\
$x_{1 f}$ & 1.286 & $x_{2 f}$ & 0 \\
$x_{3 f}$ & 0.01429 & $x_{4 f}$ & 1.865 \\
\hline
\end{tabular}

The process considered is the free-radical polymerization of methyl methacrylate in a constant volume, continuous stirred tank reactor. This example is chosen for several reasons:

1. The process is sufficiently nonlinear to require nonlinear control (Adebekun \& Schork, 1989a).

2. The process is representative of other polymerization reactors for which the development accurate nonlinear models is difficult.

3. The process model satisfies the basic assumptions in Section 2 with the exception of the system dimension. As a result, the nonlinear MRAC strategy is intractable unless the dimension of the state space is reduced.

The dimensionless model equations are (Adebekun \& Schork, 1989a),

$\frac{\mathrm{d} x_{1}}{\mathrm{~d} \tau}=x_{1 f}-x_{1}-D a_{p} W(x) x_{1} E_{x}\left(x_{2}\right)$

$\frac{\mathrm{d} x_{2}}{\mathrm{~d} \tau}=x_{2 f}-x_{2}+B D a_{p} \gamma_{p} W(x) x_{1} E_{x}\left(x_{2}\right)+\beta\left(x_{2 c}-x_{2}\right)$ $\frac{\mathrm{d} x_{3}}{\mathrm{~d} \tau}=x_{3 f}-x_{3}-D a_{d} x_{3} E_{x d}\left(x_{2}\right)$

$\frac{\mathrm{d} x_{4}}{\mathrm{~d} \tau}=x_{4 f}-x_{4}$

where $\tau$ is time, $x_{1}$ is the monomer concentration, $x_{2}$ is the reactor temperature, $x_{3}$ is the initiator concentration, $x_{4}$ is the solvent concentration, $x_{1 f}-x_{4 f}$ are the corresponding feed concentrations, $B$ is the heat of reaction, $D a_{p}$ and $D a_{d}$ are Damkohler numbers for propagation and disassociation, respectively, $\gamma_{p}$ is the activation energy for propagation, $W(x)$ is the live polymer concentration, $\beta$ is the heat transfer coefficient, and $x_{2 c}$ is the cooling jacket temperature. The solvent feed concentration $x_{4 f}$ is a function of $x_{1 f}$ and $x_{3 f}$ because the feed stream mass fractions must sum to unity. The gel effect is included in the calculation of the live polymer concentration $W(x)$ (Schmidt \& Ray, 1981). The reaction rate expressions have the form,

$E_{x}\left(x_{2}\right)=\exp \left[\frac{x_{2}}{1+\left(x_{2} / \gamma_{p}\right)}\right]$
$E_{x d}\left(x_{2}\right)=\exp \left[\frac{\gamma_{d} x_{2}}{1+\left(x_{2} / \gamma_{p}\right)}\right]$

where $\gamma_{d}$ is the activation energy for disassociation. A more complete description of the model is presented in Adebekun and Schork (1989b). The nominal operating conditions shown in Table 1 correspond to a stable equilibrium point. The objective is to control the reactor temperature $\left(y=x_{2}\right)$ by manipulating the coolant temperature $\left(u=x_{2 c}\right)$. In this study, we assume the four state variables can be measured or inferred from available on-line measurements.

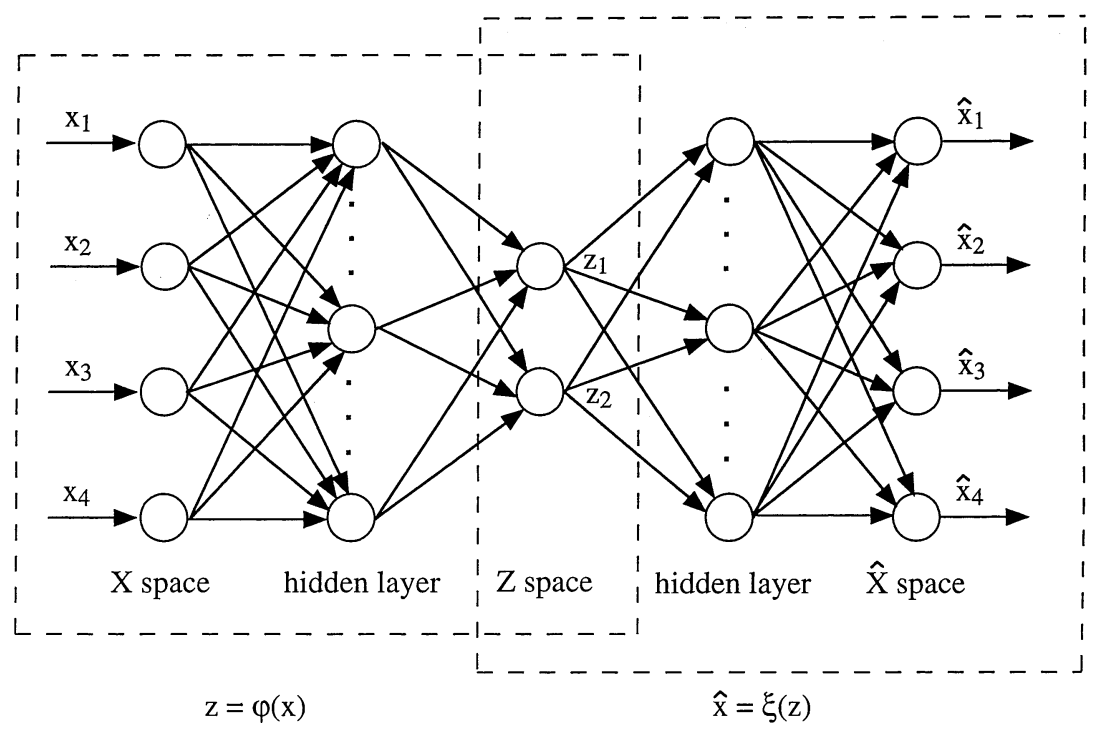

Fig. 1. Neural network for nonlinear principal component analysis. 


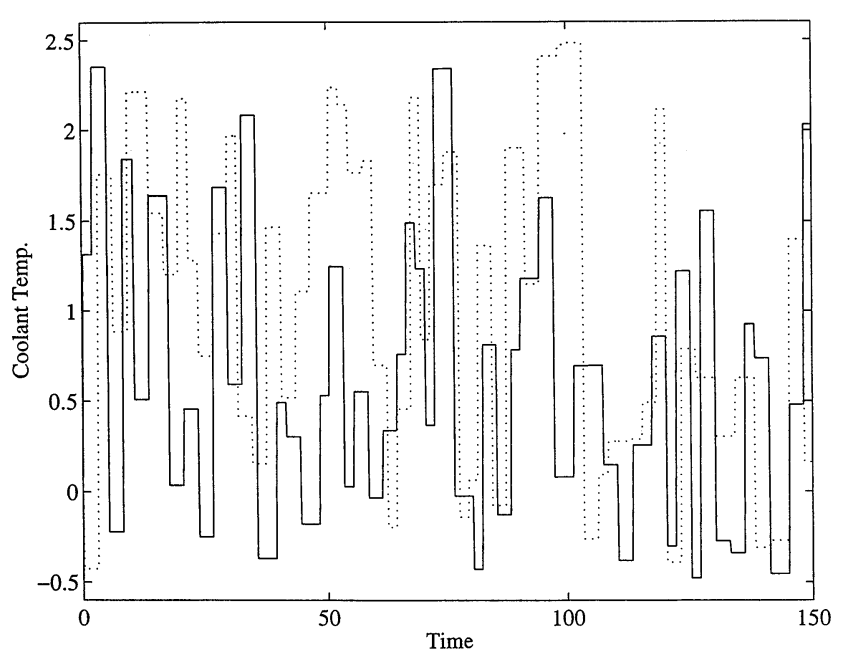

Fig. 2. Input sequences for training data set (solid) and validation data set (dot).

\subsection{Two-dimensional nonlinear MRAC controller}

The five-layer neural network used for dimensionality reduction is designed to reduce the four-dimensional state space $X$ to a two-dimensional space $Z$ (see Fig. 1). The network consists of four linear nodes in the first and fifth layer, two linear nodes in the third layer, and 15
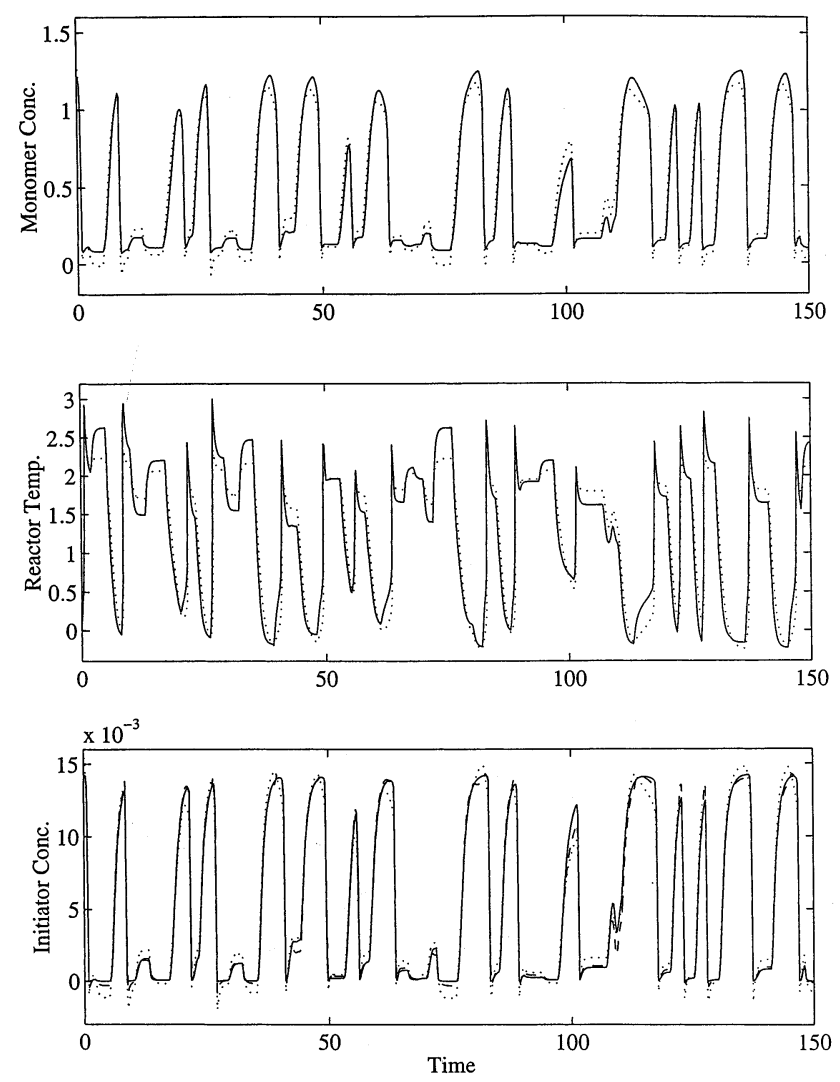

Fig. 3. Predictions for training data set: actual (solid); NPCA (dash); PCA (dot). hyperbolic activation functions in the second and fourth layers. Fig. 2 shows a random sequence of manipulated input changes used for network training. The resulting data set consists of 751 points. The network is trained over 50 epochs using the Levenberg-Marquardt backpropagation method. The predicted monomer concentration $\left(\hat{x}_{1}\right)$, reactor temperature $\left(\hat{x}_{2}\right)$, and initiator concentration $\left(\hat{x}_{3}\right)$ are compared to the actual values in Fig. 3. There are no discernible differences between the monomer concentrations and the reactor temperatures, and only very small differences between the initiator concentrations. By contrast, PCA produces linear mappings that are incapable of accurately predicting the state variable trajectories. The sum of squared errors (SSE) between the actual state variables and the estimated state variables is used as a measure of prediction accuracy. As shown in Table 2, NPCA yields a very small SSE value as compared to linear PCA.

A second random input sequence shown in Fig. 2 is used for validation. Fig. 4 shows the excellent generalization properties of the NPCA network. The prediction accuracy is similar to that obtained with the training data set. By contrast, linear PCA yields poor predictions for the validation set. These results demonstrate that NPCA is able to capture the most important process behavior by projecting the dynamics into a two-dimensional space. In general, the nonlinear MRAC strategy is applicable to systems where the critical dynamic behavior can be represented in two or (possibly) three dimensions. We envision that many processes will satisfy this requirement.

The linear controller gains $k_{1}$ and $k_{2}$ embedded in the nonlinear controller functions are computed as in (16) by constructing a linear state-space model in the reduced space $Z$. The nominal steady state in the reduced space $Z$ is computed using the mapping model $\bar{z}=\varphi(\bar{x})$. This corresponds to $\bar{z}=\left[\begin{array}{ll}1.881 & 2.052\end{array}\right]$ for the steady state in Table 1. The input sequence used for linear system identification consists of a random series of small $x_{2 c}$ changes around the nominal value (Fig. 5). The linear state-space matrices are estimated from the resulting data set of 1001 points using the MATLAB system identification toolbox:

$$
\begin{aligned}
A & =\left[\begin{array}{ll}
0.3199 & -0.5918 \\
0.8642 & -1.2752
\end{array}\right], \quad b=\left[\begin{array}{l}
0.0572 \\
0.1466
\end{array}\right], \\
c & =\left[\begin{array}{ll}
-5.5707 & 6.2331
\end{array}\right]
\end{aligned}
$$

In Fig. 5, the predicted reactor temperature generated from the linear model is compared to the actual value. The linear model captures the most important dynamic trends, but it is unable to accurately predict the nonlinear characteristics. Below we show that this degree of accuracy is sufficient for the nonlinear MRAC controller. The linear controller gains computed from (16) are $k_{1}=$ [ -4.7515 4.6977] and $k_{2}=0.5952$. 
Table 2

SSE for testing data set

\begin{tabular}{ll}
\hline Case & Training data set \\
\hline PCA & 5688 \\
Two-dimensional NPCA & 0.00016 \\
One-dimensional NPCA & 8.011 \\
\hline
\end{tabular}

The nonlinear MRAC controller utilizes a maximum of $24 \mathrm{RBFs}$ placed on a regular grid with spacing of 0.05 dimensionless units in the two-dimensional $Z$ space. The RBF scaling factors are chosen as $a_{1}=a_{2}=$ 0.2 to ensure that no more than $24 \mathrm{RBFs}$ are active at any particular time. The controller is tuned with $\gamma=$ $1.5, \eta_{1}=15$ and $\eta_{2}=5$. The $\gamma$ value corresponds to a closed-loop time constant of 0.67 dimensionless time units.

For the sake of illustration, the nonlinear MRAC controller is compared to a linear state-feedback controller designed in the $Z$ space using the empirical linear model. This comparison is meaningful because both controllers use the same plant information: the linear process model and measurements of the state variables. To include an explicit integral term for offset removal, the linear controller design is based on a second-order reference model rather than the first-order model (2).
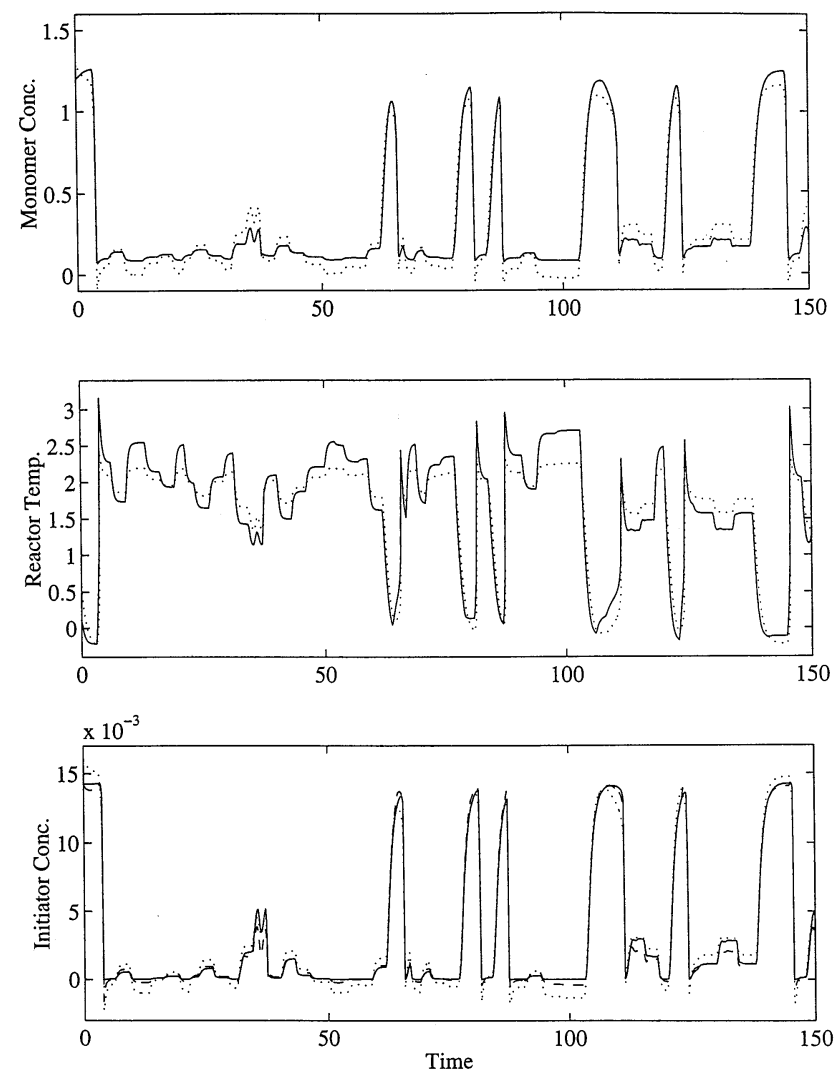

Fig. 4. Predictions for validation data set: actual (solid); NPCA (dash); PCA (dot).
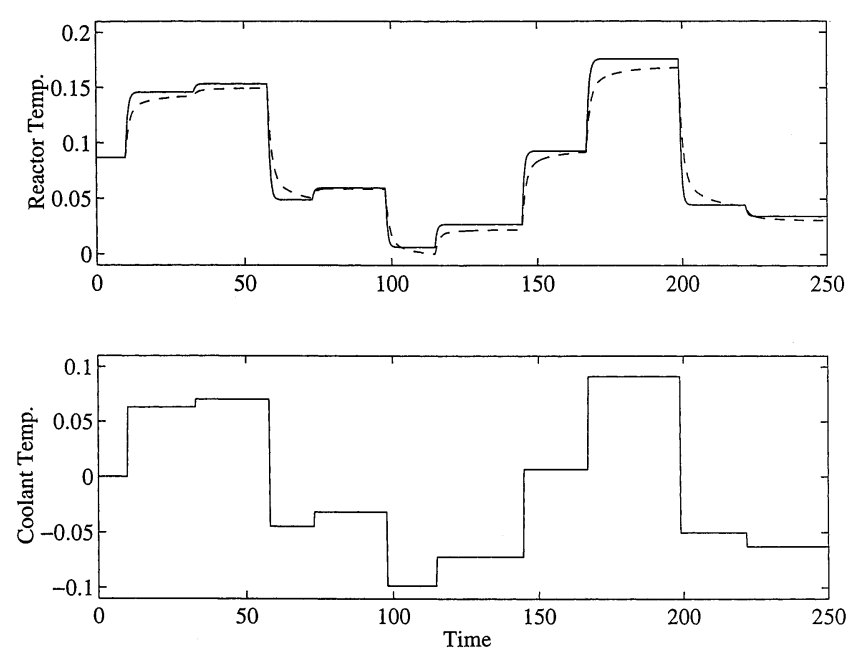

Fig. 5. Linear system identification: actual (solid); model (dash).

The resulting control law has the form,

$u^{\prime}=\frac{-c A z^{\prime}+\gamma_{1}\left(r^{\prime}-y^{\prime}\right)+\gamma_{0} \int_{0}^{t}\left(r^{\prime}-y^{\prime}\right) \mathrm{d} \tau}{c b}$

where $\gamma_{1}=3$ and $\gamma_{0}=4.5$

Fig. 6 shows the performance of the linear controller for a series of setpoint changes between three steady states where $x_{2 c}=0$. The controller has difficulty tracking the reference signal and produces undesirably large input moves. This result is attributable to the linear model used for controller design. Fig. 7 shows the nonlinear MRAC response for the same series of setpoint changes. The nonlinear controller provides very good tracking even when the steady state is in the unstable operating region. The input chattering is a general characteristic of the nonlinear MRAC controller. The adaptation gains are tuned to provide an acceptable tradeoff between the chattering and the
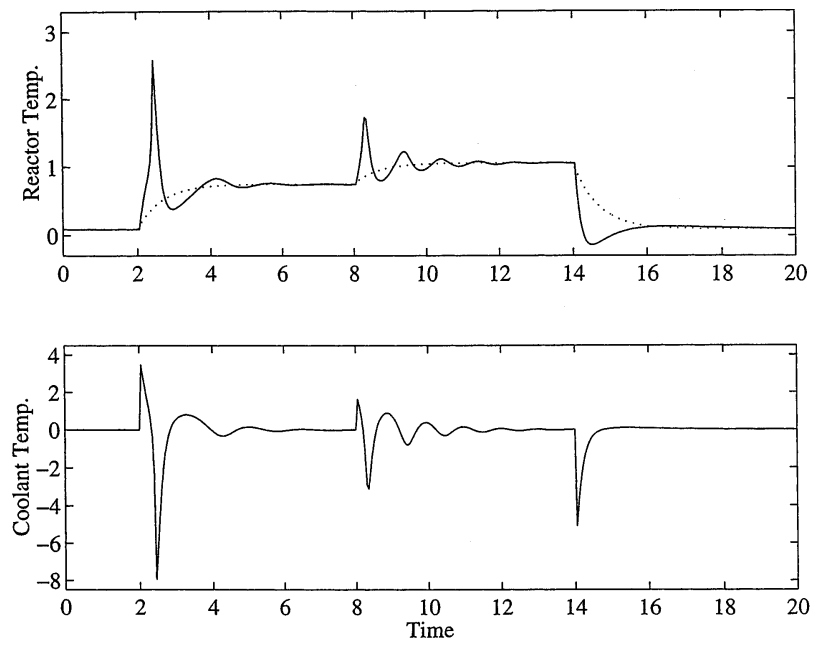

Fig. 6. Linear state-feedback controller for setpoint changes. 

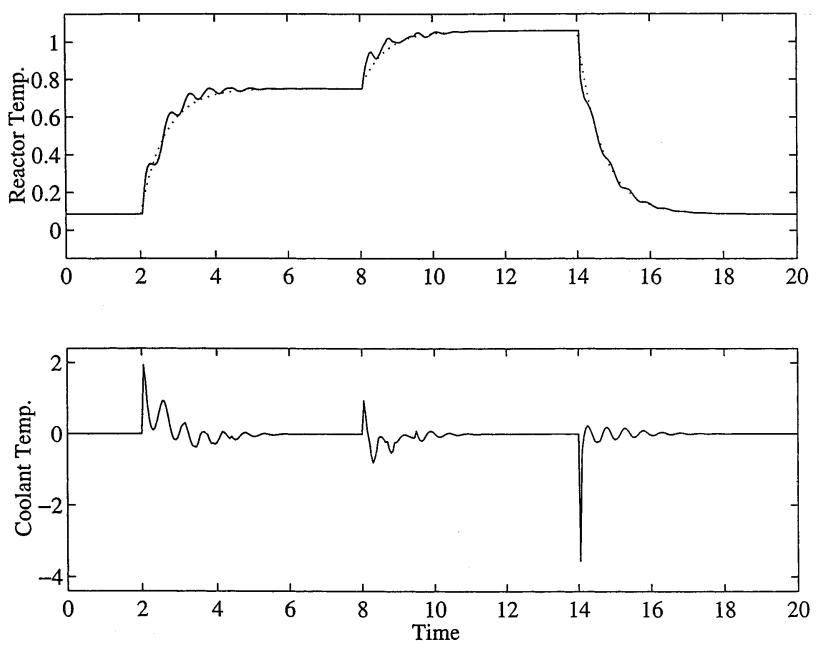

Fig. 7. Nonlinear MRAC controller for setpoint changes.
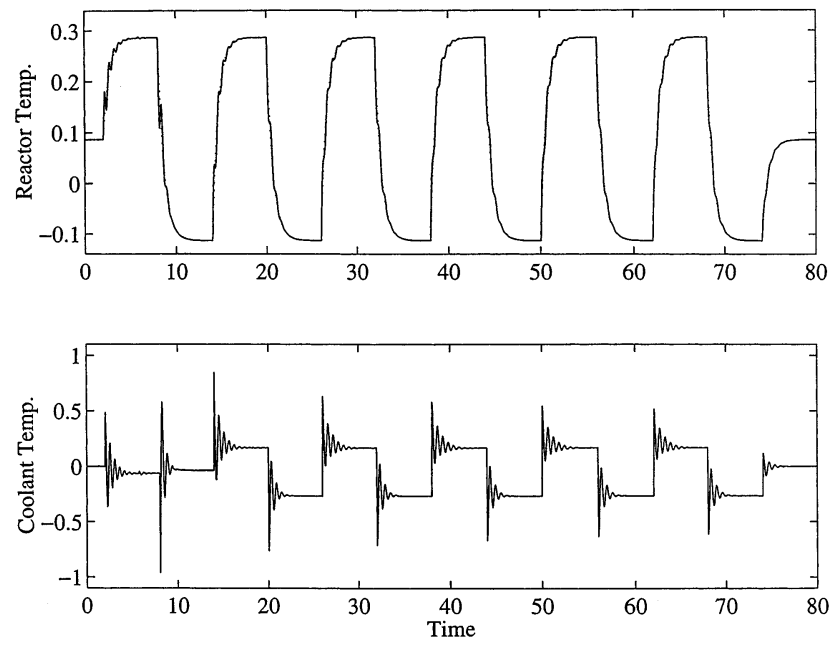

Fig. 8. Nonlinear MRAC controller for repeated setpoint changes.

speed of response. Despite this tradeoff, the manipulated input moves are well behaved as compared to the input moves of the linear controller (Fig. 6).

Fig. 8 shows the performance of the nonlinear MRAC controller for repeated setpoint changes. Because the magnitude of the setpoint change is small, the closed-loop system remains in a small region of the operating space. The controller is able to learn the appropriate control moves as there is limited on-line addition and pruning of RBFs. The tracking performance improves and the manipulated input moves become smoother as learning progresses. Learning is less pronounced when the nonlinear system operates over large regions in the reduced dimensional state space. The performance of the nonlinear MRAC controller for a random setpoint sequence is shown in Fig. 9. The controller provides excellent tracking, and the input is reasonably well behaved.
The disturbance rejection performance of the nonlinear MRAC controller for a series of feed temperature $\left(x_{2 f}\right)$ changes is shown in Fig. 10. The disturbance changes from its nominal value to 0.3 at $\tau=2$ from 0.3 to -0.3 at $\tau=8$ and from -0.3 back to its nominal value at $\tau=14$. The controller provides good regulatory performance and reasonable input moves. Fig. 11 shows the nonlinear MRAC controller response for a sequence of feed temperature disturbances of random amplitude and duration. The controller provides excellent regulatory performance.
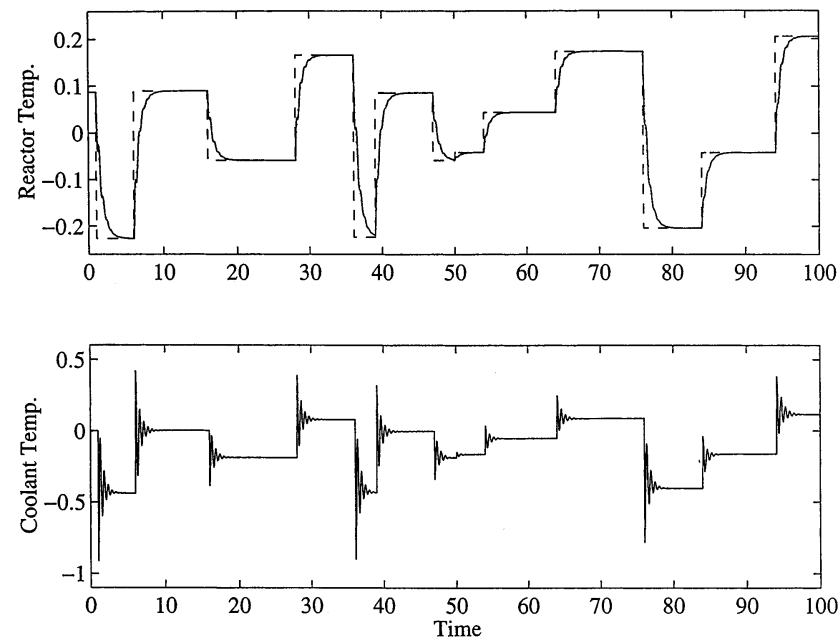

Fig. 9. Nonlinear MRAC controller for random setpoint changes.
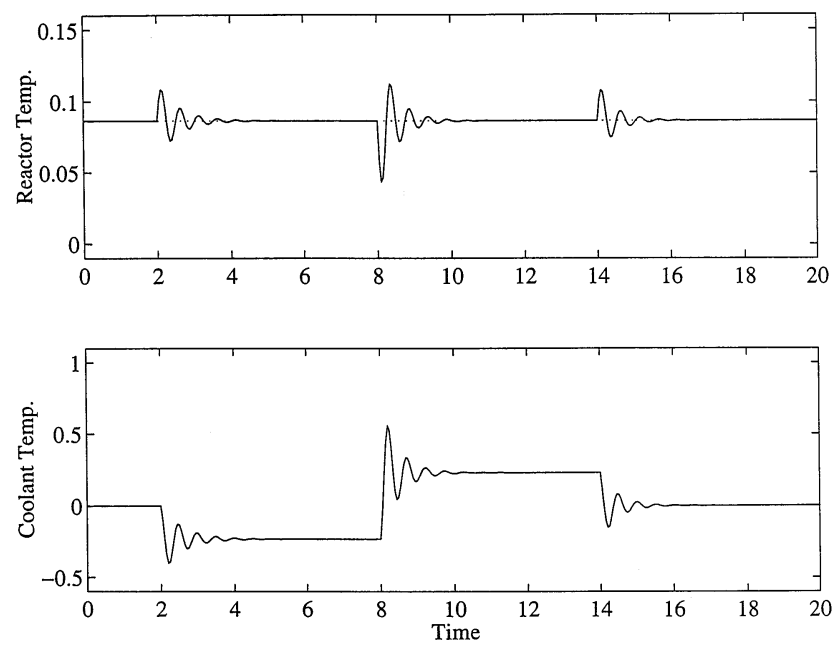

Fig. 10. Nonlinear MRAC controller for unmeasured disturbance changes. 

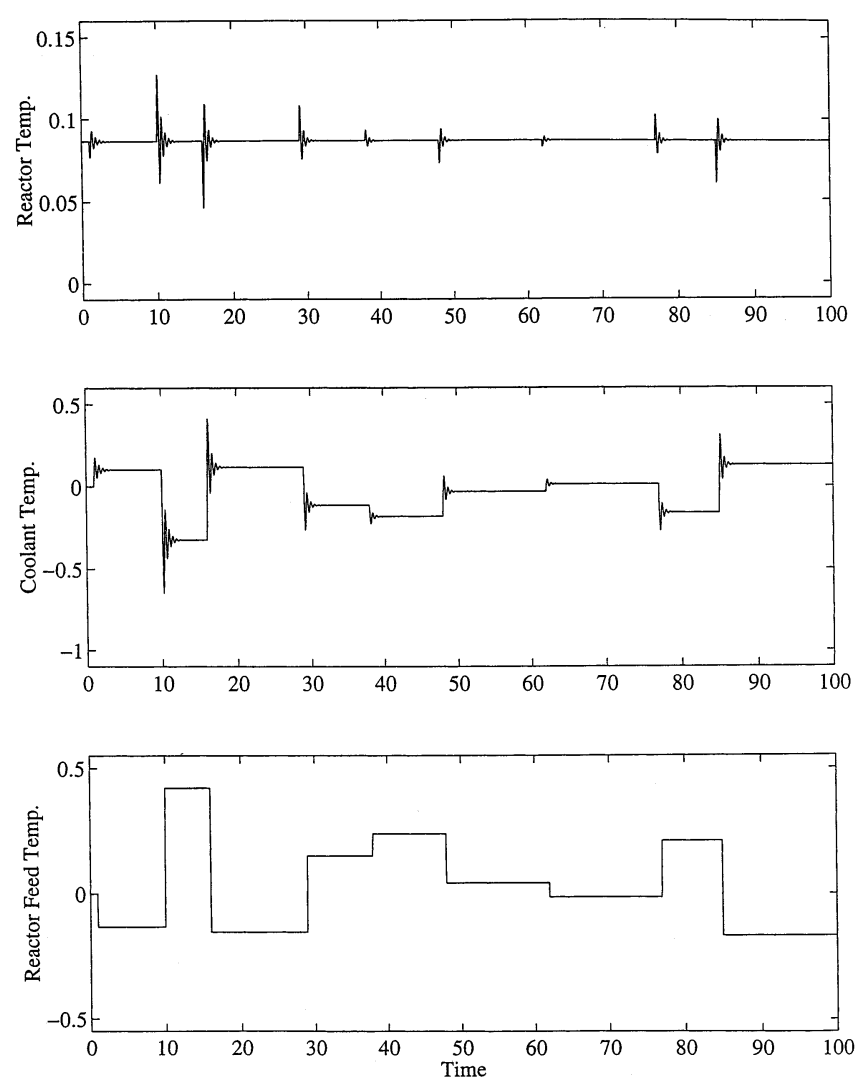

Fig. 11. Nonlinear MRAC controller for random unmeasured disturbance changes.
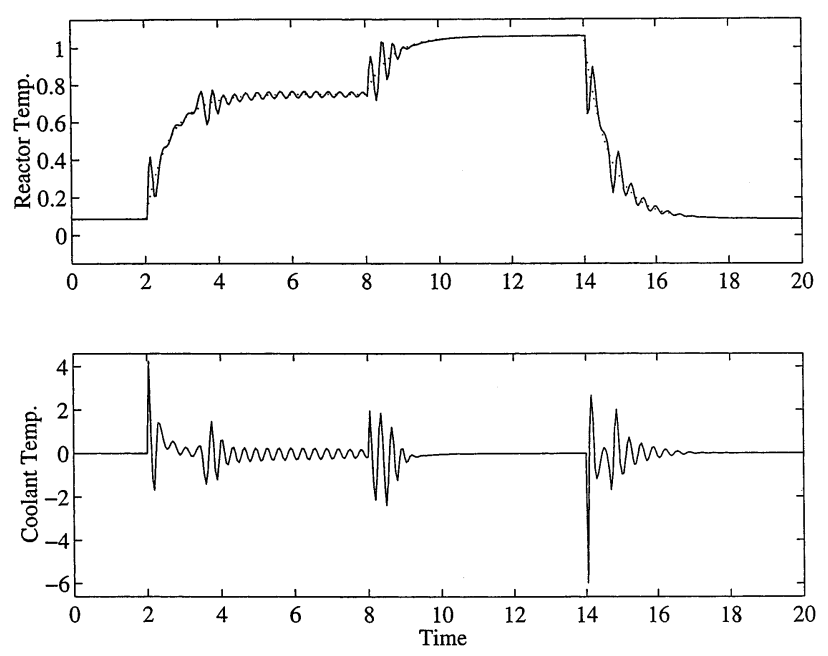

Fig. 12. One-dimensional nonlinear MRAC controller for setpoint changes.

\subsection{One-dimensional nonlinear MRAC controller}

The previous simulation results show the performance of the nonlinear MRAC controller when the state space is reduced from four dimensions to two dimensions. Now we consider reducing the original state space $X$ to a one-dimensional space $Z$. The objective of this test is to demonstrate the tradeoff between prediction accuracy of the NPCA network and the computational efficiency of the resulting nonlinear MRAC controller. A five-layer neural network with a single linear node in the third layer and the same number of nodes in the other layers is trained over 50 epochs using the random input sequence in Fig. 2. The prediction accuracy is degraded as compared to the two-dimensional case.

The linear controller gains $k_{1}=-1.8724$ and $k_{2}=$ 0.2714 are computed using the nominal operating point $\bar{z}=-0.0651$ The nonlinear MRAC controller utilizes 10 RBFs instead of the 24 RBFs used in the two-dimensional case. This reduction in the number of RBFs employed provides a significant enhancement in computational efficiency. The regular grid for RBF center placement has a spacing of 0.05 dimensionless units. The scaling factor is chosen as $a=0.25$ to ensure that a maximum of ten RBFs are active at any particular time. The controller is tuned with $\gamma=1.5, \eta_{1}=25$ and $\eta_{2}=0.2$.

Fig. 12 shows the performance of the nonlinear MRAC controller for the one-dimensional case using the same setpoint sequence as in Fig. 7. As compared to the two-dimensional case (Fig. 7), the setpoint tracking performance is poor and the input moves are quite oscillatory. The poor performance of the one-dimensional controller is attributable to two factors. First, the linear model used to construct the nonlinear MRAC controller is unable to capture the dominant process dynamics when restricted to a single dimension (not shown). More importantly, a significant amount of information is lost when NPCA is used to contruct a one-dimensional $Z$ space. This example shows the type of prediction and control results that are obtained when the dimension of the reduced space is not sufficiently large.

\subsection{Output feedback nonlinear MRAC controller}

A fundamental assumption used in the development of the nonlinear MRAC strategy is that all the state variables can be measured or estimated from available measurements. In many applications, only a subset of the state vector is available for feedback control. The output feedback problem is investigated for the polymerization reactor example to demonstrate possible extensions of the controller design procedure. We assume the monomer concentration $\left(x_{1}\right)$ and reactor temperature $\left(x_{2}\right)$ are measured and the initiator concentration $\left(x_{3}\right)$ and solvent concentration $\left(x_{4}\right)$ are unmeasured. It is important to emphasize that nonlinear observer design is not feasible because an explicit nonlinear model is unavailable. As an alternative, the nonlinear MRAC controller is constructed directly in the two-dimensional measured space. This approach can be interpreted as restricting the $\varphi(x)$ and $\xi(z)$ 

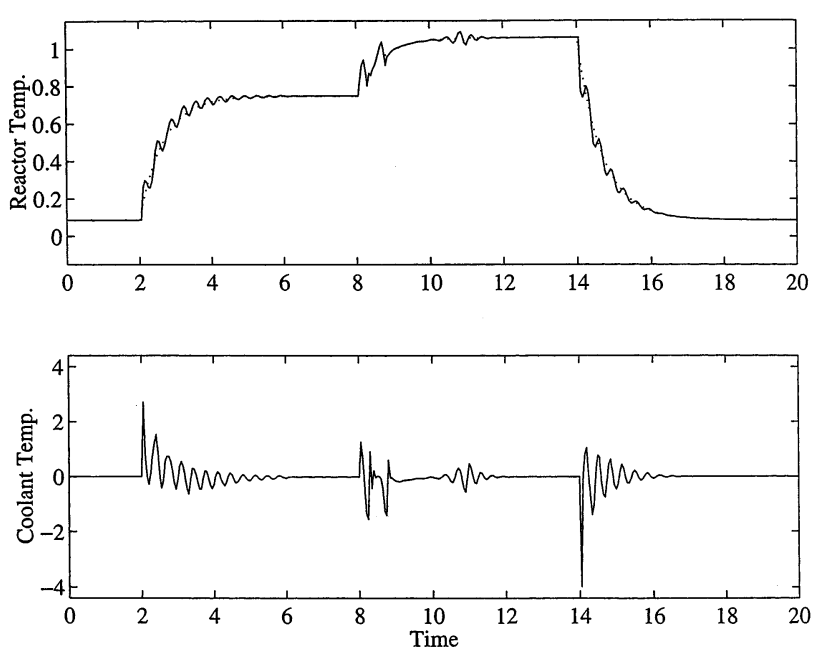

Fig. 13. Output feedback nonlinear MRAC controller for setpoint changes.

mappings such that the reduced dimensional space is equal to the measurement space. For the reactor example, the dimension of the measurement space is sufficiently small to allow direct application of the nonlinear MRAC strategy. The use of NPCA to generate a lower dimensional measured space may be required for more complicated processes.

The nonlinear MRAC design begins with determination of the appropriate linear controller gains. A random input sequence about the nominal steady state $\bar{z}=\left[\begin{array}{ll}1.2057 & 0.08653\end{array}\right]$ is used to generate input/output data for linear system identification. The linear controller gains computed from (16) are $k_{1}=$ $\left[\begin{array}{ll}-0.2210 & 0.9021]\end{array}\right.$ and $k_{2}=0.4626$. The nonlinear MRAC controller utilizes $24 \mathrm{RBFs}$ placed on a regular grid with spacing of 0.05 dimensionless units in the two-dimensional measurement space. The RBF scaling factors are chosen as $a_{1}=a_{2}=0.2$ to ensure that a maximum of $24 \mathrm{RBFs}$ are active at any particular time. The controller is tuned with $\gamma=1.5, \eta_{1}=25$ and $\eta_{2}=2$.

Fig. 13 shows the tracking performance of the nonlinear MRAC controller for the same setpoint sequence as that in Fig. 7. The controller performs reasonably well despite lacking measurements of the initiator and solvent concentrations. The performance is better than that obtained for the one-dimensional state-feedback controller (Fig. 12) and is comparable with that obtained for the two-dimensional state-feedback controller (Fig. 7). Although not shown here, the output feedback controller yields acceptable regulatory performance for the feed temperature disturbance tests shown in Fig. 10 and Fig. 11. These results indicates that the measured state variables capture most of the important process dynamics. As a result, the lack of initiator and solvent concentration measurements does not result in a significant loss of closed-loop performance. It is important to note that these results are highly problem specific. We anticipate that nonlinear MRAC with fullstate feedback will yield superior performance in most applications.

\section{Summary and conclusions}

A nonlinear model reference adaptive control strategy in which nonlinear principal component analysis is used to reduce the system dimension has been developed and evaluated via simulation. The proposed method utilizes radial basis function (RBF) networks to approximate unknown functions in the associated input-output linearizing controller. Computational efficiency is enhanced by using nonlinear principal component analysis to reduce the dimension of the state space. This modification facilitates on-line construction of the nonlinear controller because basis functions can be placed in a lower dimensional space. A second computational enhancement is the development of a novel RBF center placement algorithm that reallocates a fixed number of basis functions to continuously track the current operating point in the reduced dimensional state space. This modification eliminates computational problems associated with the number of basis functions increasing with time. The proposed controller design method has been evaluated using a four-dimensional polymerization reactor model. The simulation results demonstrate that the computational enhancements enable the nonlinear model reference adaptive control strategy to be successfully applied to nonlinear processes of moderate complexity.

\section{Acknowledgements}

Financial support from an NSF Career Development Award (CTS-9501368) and the DuPont Company is gratefully acknowledged.

\section{References}

Adebekun, D. K., \& Schork, F. J. (1989a). Continuous solution polymerization reactor control. 1. Nonlinear reference control of methyl methacrylate polymerization. Industrial Engineering Chemistry Research, 28, 1308-1324.

Adebekun, D. K., \& Schork, F. J. (1989b). Continuous solution polymerization reactor control. 2. Estimation and nonlinear reference control during methyl methacrylate polymerization. Indus trial Engineering Chemistry Research, 28, 1846-1861.

Chen, S., Billings, S. A., \& Grant, P. M. (1992). Recursive hybrid algorithm for non-linear system identification using radial basis functions. International Journal of Control, 55, 1051-1070.

Dong, D., \& McAvoy, T. J. (1994). Nonlinear principal component analysis - based on principal curves and neural networks. In Proceedings of the American Control Conference (pp. 1284-1288). Baltimore, MD, USA. 
Hastie, T., \& Stuetzle, W. (1989). Principal curves. Journal of the American Statistical Association, 84, 502-516.

Isidori, A. (1989). Nonlinear control systems. New York: Springer.

Kramer, M. A. (1991). Nonlinear principal component analysis using autoassociative neural networks. American Institute of Chemical Engineers Journal, 37, 233-243.

Kresta, J. V., MacGregor, J. F., \& Marlin, T. E. (1991). Multivariate statistical monitoring of process operating performance. Canadian Journal of Chemical Engineering, 69, 35-47.

Ljung, L. (1989). System identification: theory for the user. Engelwood Cliffs, NJ: Prentice-Hall.

Mardia, K., Kent, J., \& Bibby, J. (1980). Multivariate Analysis. London: Academic Press.

McLain, R. B., \& Henson, M. A. (1997). Nonlinear model reference adaptive control with embedded linear models. In American Institute of Chemical Engineers Annual Meeting. Los Angeles, CA.

McLain, R. B., Henson, M. A., \& Pottmann, M. (1999). Direct adaptive control of partially known nonlinear systems. IEEE Transactions on Neural Networks, 10, 714-721.

Pearson, R. K., \& Ogunnaike, B. A. (1997). Nonlinear process identification. In M. A. Henson, \& D. E. Seborg, Nonlinear process control (pp. 11-110). Englewood Cliffs, NJ: Prentice-Hall.
Polycarpou, M. M., \& Ioannou, P. A. (1991). Identification and control of nonlinear systems using neural network models: Design and stability analysis. Technical Report MC-2563, University of Southern California.

Pottmann, M., \& Henson, M. A. (1997). Compactly supported radial basis functions for adaptive process control. Journal of Process Control, 7, 345-356.

Sanner, R. M., \& Slotine, J. -J. E. (1992). Gaussian networks for direct adaptive control. IEEE Transactions of Neural Networks, 3, $837-863$.

Sastry, S., \& Bodson, M. (1989). Adaptive control: stability, convergence, and robustness. New York: Prentice-Hall.

Sastry, S., \& Isidori, A. (1989). Adaptive control of linearizable systems. IEEE Transactions of Automatic Control, AC-34, 11231131 .

Schmidt, A. D., \& Ray, W. H. (1981). The dynamic behavior of continuous polymerization reactors - I. Chemical Engineering Science, 36, 1401-1410.

Tan, S., \& Mavrovouniotis, M. L. (1995). Reducing data dimensionality through optimizing neural network inputs. American Institute of Chemical Engineers Journal, 41, 1471-1480. 